\title{
Mecanismes De Contamination Des Eaux Souterraines Dans Le Secteur Du Lac Mbeubeuss, Dakar, Senegal
}

\author{
Essouli Olivier Florent, Docteur, Maître-Assistant
}

Université Marien NGOUABI, Faculté des Sciences et Techniques,

Laboratoire de Géosciences, Brazzaville, Congo

Gladima-Siby Sophie Aïssatou, Docteur, Maître-Assistant

Université Cheikh Anta Diop, Faculté des Sciences et Techniques,

Département de Géologie, Dakar-Fan, Sénégal

Miyouna Timothée, Docteur, Maître-Assistant

Université Marien NGOUABI, Faculté des Sciences et Techniques,

Laboratoire de Géosciences, Brazzaville, Congo

Matini Laurent, Docteur, Maître de Conférences

Université Marien NGOUABI, Ecole Normale Supérieure, Laboratoire de

Chimie, Brazzaville, Congo

Faye Serigne, Professeur Titulaire

Université Cheikh Anta Diop, Faculté des Sciences et Techniques,

Département de Géologie, Dakar-Fan, Sénégal

Doi: 10.19044/esj.2018.v14n27p254 URL:http://dx.doi.org/10.19044/esj.2018.v14n27p254

\begin{abstract}
The physicochemistry of groundwater in the immediate environment of the dump, and its surroundings, shows that the true value of the electrical conductivity of groundwater is close to $2000 \mu \mathrm{S} / \mathrm{cm}$. The value of electrical conductivity that is greater than $2000 \mu \mathrm{S} / \mathrm{cm}$ demonstrates groundwater contamination through the Mbeubeuss Lake landfill, with chemical groundwater facies dominated by Sodium Chloride, Potassium Chloride, and Calcium Chloride. The study of the unsaturated zone of the aquifer and the relationship between the major chemical elements of groundwater, rainwater, and seawater made it possible to specify, on one hand, the sources of mineralization of the groundwater at the Mbeubeuss Lake site and its surroundings. Indeed, the situation close to the sea would suggest a considerable intake of salts by aerosols and sea spray. Based on contribution to this study, the influence of the old sediments of the dry Lake Mbeubeuss and the percolation of leachates resulting from the decomposition of garbage landfilled under the action of rainwater would be added. On the other hand,
\end{abstract}


this study also made it possible to determine the main processes of controlling the mineralization of groundwater. This includes the marine inputs, the dissolution-precipitation of clay minerals present in the geological formations constituting the aquifer, the dilution- concentration, evaporation, and anthropogenic pollution.

Keywords: Discharge, hydrochimy, mineralization, pollution, ground waters

\section{Resumen}

La physico-chimie des eaux souterraines dans l'environnement immédiat de la décharge publique et de ses environs, montre que la véritable valeur de la conductivité électrique des eaux souterraines est voisine de 2000 $\mu \mathrm{S} / \mathrm{cm}$. Cependant, les valeurs de conductivité électrique supérieures à 2000 $\mu \mathrm{S} / \mathrm{cm}$ font preuve de la contamination des eaux souterraines par la décharge publique du lac Mbeubeuss, les faciès chimiques des eaux souterraines étant dominés par le chloruré sodique et potassique et le chloruré calcique. L'étude de la zone non saturée de l'aquifère et de la relation entre les éléments chimiques majeurs des eaux souterraines, des eaux de pluies et de l'eau de mer, a permis de préciser d'un côté les sources de minéralisation des eaux souterraines au site du lac Mbeubeuss et de ses environs. En effet, la situation à proximité de la mer laisserait supposer un apport considérable de sels par les aérosols et embruns marins. A cet apport s'ajouteraient l'influence des anciens sédiments du lac Mbeubeuss asséché et la percolation des lixiviats issus de la décomposition des ordures mises en décharge sous l'action des eaux de pluie. De l'autre côté, cette étude a aussi fait possible de déterminer les principaux processus contrôlant la minéralisation des eaux souterraines, lesquels sont les apports marins, la dissolution-précipitation des minéraux argileux présents dans les formations géologiques constituant l'aquifère, la dilution-concentration, l'évaporation et la pollution anthropique.

Mots-clés : Décharge, hydrochimie, zone non saturée, pollution, eaux souterraines

\section{Introduction}

Les eaux souterraines sont d'une importance capitale dans la plupart des régions du monde, raison pour laquelle sa disponibilité, son accessibilité et son exploitabilité sont des objectifs majeurs des projets de développement à niveau mondiale (Kouassi et al., 2011).

De la même manière, l'accès à l'eau potable par les populations constitue l'un des grands problèmes auxquels sont confrontés les gouvernements du monde. Il est donc essentiel de mobiliser des ressources pour développer les infrastructures hydrauliques dans les zones rurales et 
urbaines car l'eau, qui constitue un élément incontournable pour le développement économique, est menacée de contamination. Il convient donc de connaitre et suivre la qualité de cette ressource (Eblin et al., 2014).

Les enjeux environnementaux et sanitaires liés aux pollutions toxiques dans les milieux aquatiques sont au cœur de nombreux débats de société, et la prise de conscience de la nécessité de réduire la pollution toxique est de plus en plus importante. En effet, les polluants toxiques ont des impacts non seulement sur l'environnement, mais aussi sur la santé humaine. Les conséquences sanitaires peuvent être importantes, voire même mortelles. Par exemple, dans les années 1950-60 au Japon, une pollution au cadmium dans les rizières a déclenché de nombreux troubles rénaux et osseux, entraînant une importante mortalité dans les populations de ces régions (Boucheseiche et al., 2002).

Par ailleurs, les coûts économiques de la dépollution sont importants (construction de stations d'épuration pour les industries, mise au point de nouveaux procédés, décontamination des sites pollués et des sols pollués). Ces coûts peuvent être aussi indirects en empêchant l'approvisionnement en eau potable des populations.

Dans le monde, plus de 2,3 millions d'habitants sont atteints de maladies hydriques. La fourniture d'eau potable et la mise en place de bons dispositifs d'assainissement auraient d'importantes retombées sur la santé des populations. Par ailleurs, des études ont montré que ces dispositions réduiraient la mortalité de 1'ordre de 2,1 millions de morts à la suite de maladies diarrhéiques, 150 millions pour les cas de schistosomiase et 75 millions pour les maladies du trachome (Esrey et al., 1991; OMS, 1997; Hinrichsen et al., 1998).

En 1991, les études des impacts de cette décharge sur 1'environnement, commandées par le Ministère Sénégalais de l'Environnement ont montré que celle-ci renfermait un fort potentiel de contamination et, dès lors, constituait un danger pour la population et l'environnement. Dans cette optique, la présente étude se fixe pour principal objectif de préciser les principaux mécanismes de contamination des eaux souterraines par la décharge publique dans le secteur de Thiaroye/lac Mbeubeuss.

\section{Matériel et Méthodes}

\section{2-1. Contexte général de la région d'étude}

La région de Dakar représente l'extrême pointe occidentale de l'Afrique de l'ouest et l'extrême ouest du Sénégal. Le secteur de Thiaroye/lac Mbeubeuss est situé à une trentaine de kilomètres de la ville de Dakar, entre les latitudes $14^{\circ} 47^{\prime}$ et $14^{\circ} 50^{\prime}$ Nord et les longitudes $17^{\circ} 16^{\prime}$ et $17^{\circ} 20^{\prime}$ Ouest tel que présenté à la Figure 1. 


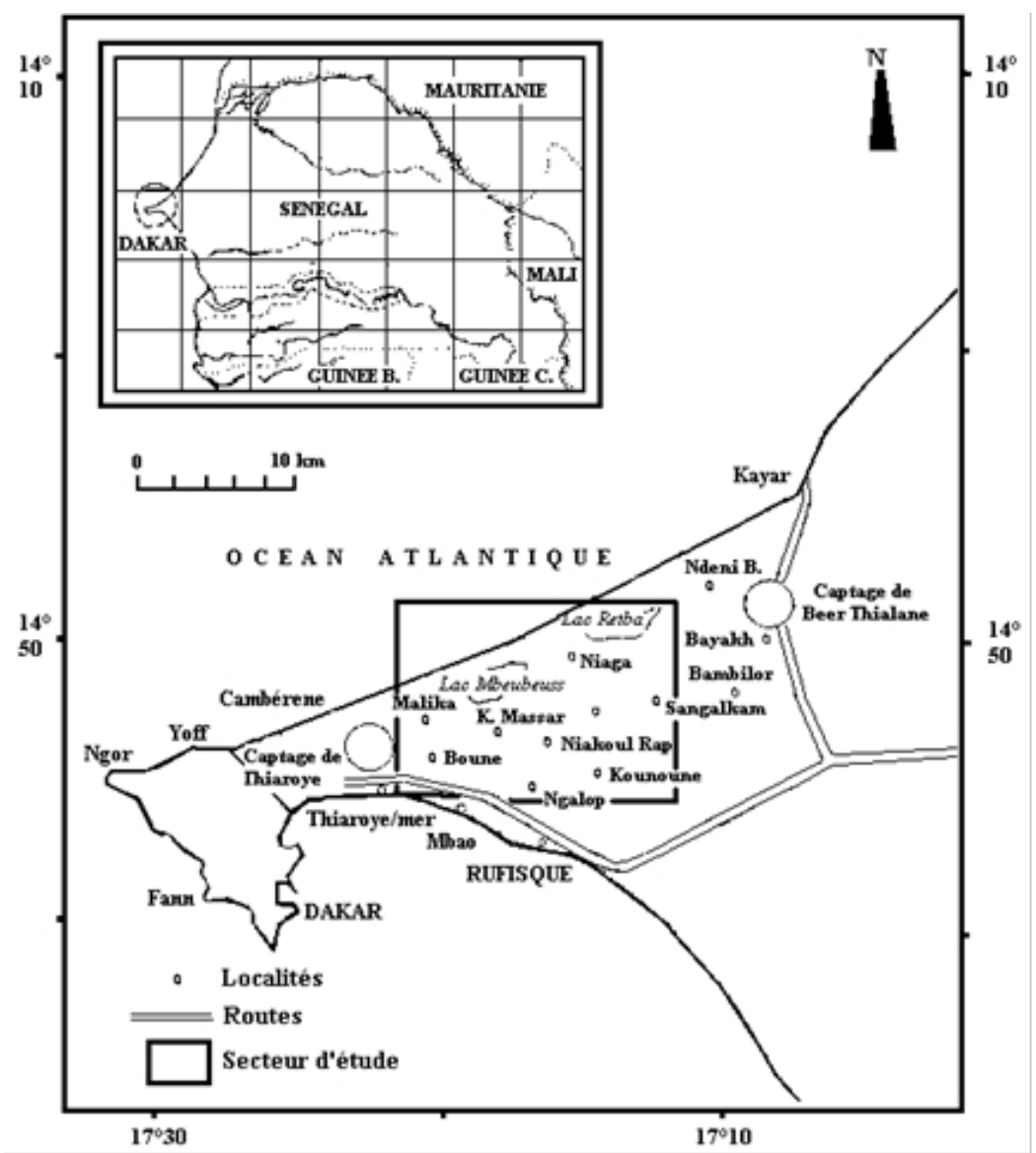

Figure 1. Situation du secteur de Thiaroye/lac Mbeubeuss

Dans ce secteur se trouve une dépression du lac Mbeubeuss asséché inondable pendant la saison des pluies, où est implantée la décharge publique d'ordures ménagères et des déchets des hôpitaux, des laboratoires des universités et des industries de la région dakaroise et de ses environs. La superficie du lac Mbeubeuss est de 600 ha environ et actuellement la décharge publique occupe moins de $25 \%$ de cette superficie et évolue beaucoup plus en hauteur.

A l'origine le site du lac Mbeubeuss n'était destiné qu'à recevoir les refus issus de l'usine de compostage de Mbao qui a fonctionné de 1968 à 1970. C'est avec la fermeture de la décharge publique de Hann en 1970 que le site du lac Mbeubeuss a été finalement transformé en décharge publique et reçoit depuis cette date la quasi-totalité des déchets collectés dans l'agglomération dakaroise. Pourtant, cette décision a été prise sans la réalisation d'aucune étude préalable d'implantation destinée à apprécier l'aptitude du site à l'exploitation d'une décharge. L'environnement hydrogéologique du site du lac Mbeubeuss (zone des Niayes, où la nappe est affleurante à sub-affleurante), le matériel 
géologique de l'aquifère constitué essentiellement par des sables favorisant un transfert rapide des polluants d'une part, et l'exploitation anarchique de cette décharge, la diversité et le volume des déchets stockés (matières de vidange des voitures, ordures des hôpitaux et des laboratoires des universités, les ordures ménagères) d'autre part, pourraient constituer une source potentielle de pollution de la nappe phréatique par lessivage des déchets stockés sous l'action des eaux de pluie.

La région de Dakar appartient au bassin sénégalo-mauritanien, le plus vaste des bassins côtiers existants au niveau de la marge nord-ouest de l'Afrique. Sa structure est simple et caractérisée par un socle qui s'enfonce d'est en ouest. Sur le plan structural, la région de Dakar a été affectée par des failles verticales de directions NE-SW à NNE-SSW qui sont à l'origine de la mise en place des horsts de Dakar et de Ndiass et du graben de RufisqueSangalkam (Bellion, 1987).

La stratigraphie locale est connue grâce aux forages pétroliers et hydrauliques et met en évidence des formations datées du Crétacé supérieur (Maastrichtien) au Quaternaire (Figure 2) dont seules les formations du Quaternaire présentent un intérêt hydrogéologique (Faye, 1994).

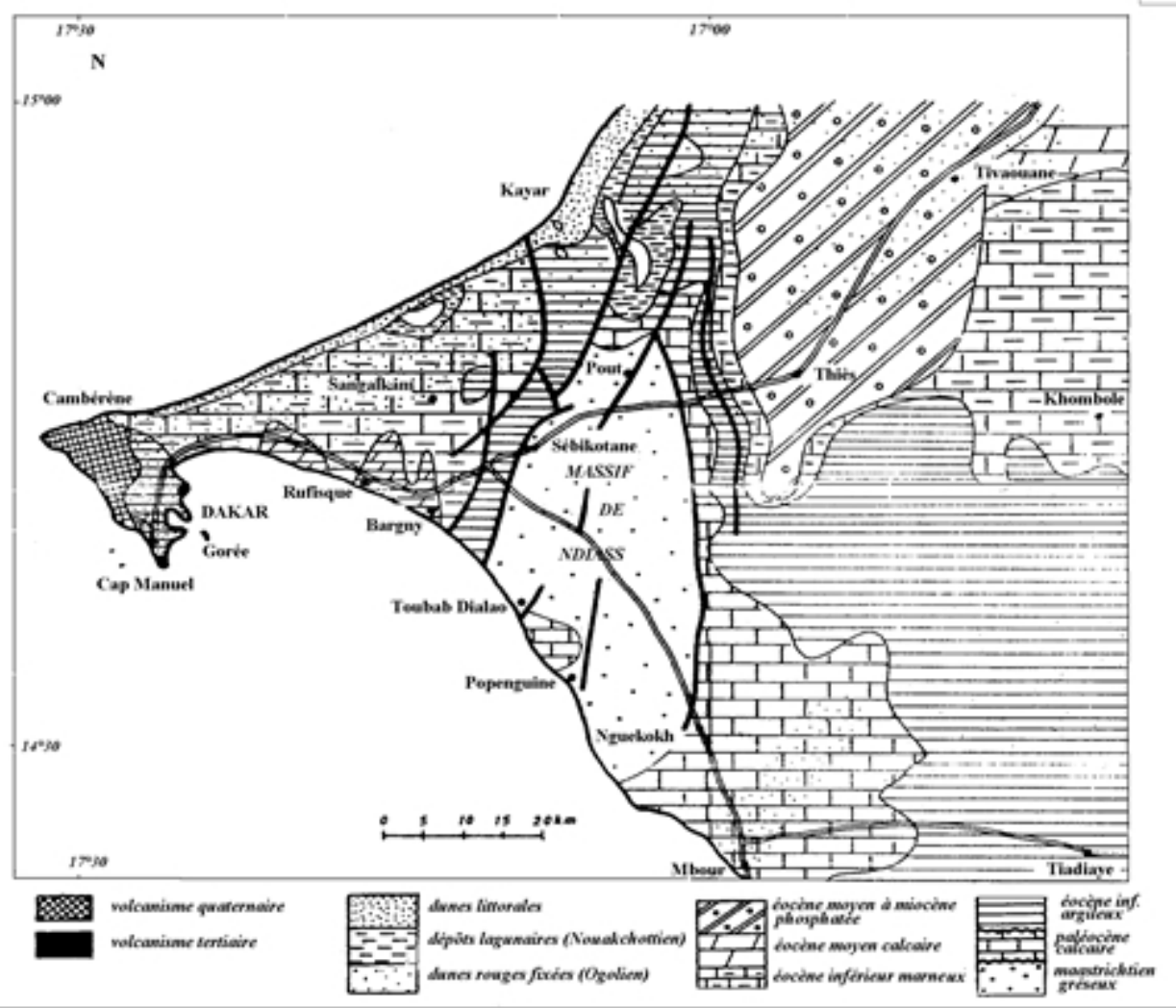

Figure 2. Carte géologique des régions de Dakar et de Thiès (Faye, 1994) 
Le système aquifère des sables quaternaires s'étend de la grande Niaye de Pikine jusqu'au lac Tanma. Les sables quaternaires reposent sur un substratum marno-argileux du Tertiaire, dont la configuration va influencer l'écoulement des eaux souterraines. Par ailleurs, l'interruption de la sédimentation des dépôts sableux par la formation de sables argileux et argiles sableuses issues des transgressions marines fait que l'aquifère des sables quaternaires soit subdivisé en plusieurs couches. L'aquifère en totalité libre présente quelques poches très limitées de semi captive. La morphologie du mur de l'aquifère est relativement tourmentée. Globalement, le toit des marnes s'enfonce vers la côte nord dans la zone de Thiaroye /lac Mbeubeuss et vers le lac Retba, alors qu'il remonte entre le Cap des Biches et Niakoul Rap (Faye, 1994 ; Martin, 1970).

L'épaisseur des sables aquifères est très variable, suivant les variations de la morphologie du substratum. Les épaisseurs importantes des sables mouillés dans les dépressions modelées par l'érosion suivent les grandes lignes tectoniques et on observe des vallées fossiles et des cuvettes lacustres dont les plus importantes, la cuvette de Thiaroye et la dépression de Beer Thialane, constituent les principaux centres de captages pour l'exploitation de cet aquifère (Martin, 1970).

Du fait de la grande variabilité de la géométrie de l'aquifère des sables quaternaires, les paramètres hydrodynamiques présentent également une variabilité spatiale. Les transmissivités varient de 2,45 à $6,75.10^{-4} \mathrm{~m}^{2} / \mathrm{s}$, les perméabilités de l'ordre de 0,40 à $7,40.10^{-4} \mathrm{~m} / \mathrm{s}$ et le coefficient d'emmagasinement allant de 1,06 à 2,51\% (Cissé Faye, 2000).

La piézométrie dans le secteur de Thiaroye/lac Mbeubeuss dressée à partir des données de mars 2003, montre un sens d'écoulement global en direction de l'océan, des lacs intérieurs (Mbeubeuss et Retba) et des dépressions piézométriques (Figure 3). Cet écoulement est conditionné par la remontée du substratum marno-calcaire de l'Eocène vers le sud-est, conséquence des mouvements tectoniques ayant conduit à la mise en place des horsts de Dakar et de Ndiass et du graben de Rufisque-Bargny (Martin, 1970). 


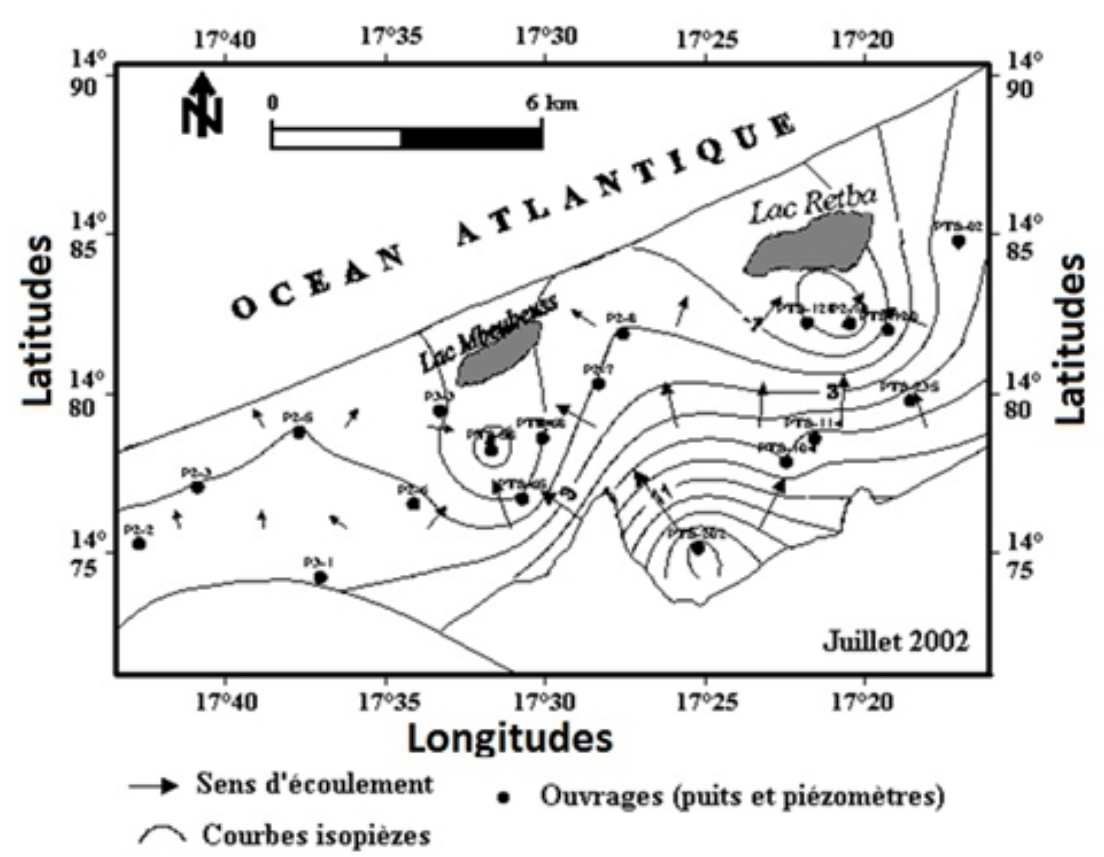

Figure 3. Piézométrie de l'aquifère dans le secteur du lac Mbeubeuss (Essouli, 2005).

La distribution des niveaux piézométriques dans le secteur de Thiaroye/lac Mbeubeuss, se caractérise par la présence d'une aire d'alimentation de l'aquifère des sables quaternaires (ou dôme piézométrique) aux puits (Pts-202).

On remarque aussi l'existence de deux aires de vidange de la nappe des sables quaternaires (ou dépressions piézométriques), l'une englobant les puits (Pts-58, Pts-65 et Pts-68) dans la zone du lac Mbeubeuss / Keur Massar et l'autre autour des points d'eau (Pts-120, Pts-128 et P2-10) dans la zone du lac Retba. Les gradients hydrauliques sont généralement faibles et ne dépassent guère 1\% dans le secteur étudié (Essouli, 2005).

\section{2-2. Acquisition des données}

De nombreux points d'eau (forages, piézomètres et puits villageois) exploitant l'aquifère des sables quaternaires de Thiaroye / lac Mbeubeuss ont été réalisés suite aux différents programmes d'études concernant l'alimentation en eau potable de la région de Dakar. Ces points d'eau sont placés sous la responsabilité de la Direction de Gestion et Planification des Ressources en Eau (DGPRE) du Ministère Sénégalais de l'Hydraulique (Forages et piézomètres) et des maraîchers (Puits villageois).

Du 22 au 24 juillet 2002 sur le terrain, des échantillons de sols ont été prélevés à l'aide d'une tarière manuelle de type australien (avec des tubes de rajout de 1 à 1,5 m) de la surface du sol jusqu'à la nappe. Des profils de moins 
de $3 \mathrm{~m}$ de profondeur de la surface du sol jusqu'à la nappe ont été réalisés dans la zone non saturée de l'aquifère des sables quaternaires en juillet 2002, en début de l'hivernage où les phénomènes évaporatoires sont intenses. Ces profils (PMB-1, PMB-3, PMB-4 et PMB-6) sont situés sur l'axe des piézomètres (PZ-2 et PZ-3) du site du lac Mbeubeuss (Figure 4).

Le pas de prélèvement est de $0,25 \mathrm{~m}$. Les niveaux statiques de la nappe et les hauteurs des margelles par rapport au sol des piézomètres d'observation et puits traditionnels ont été mesurés à l'aide d'une sonde lumineuse et sonore. Parallèlement au suivi piézométrique, des paramètres physiques in-situ (température, $\mathrm{pH}$ et conductivité électrique) ont été mesurés et des échantillons d'eaux prélevés dans des bouteilles en polyéthylène bien étanches pour des fins d'analyses chimiques au laboratoire d'hydrologie et d'hydrochimie du département de Géologie de la Faculté des Sciences et Techniques de l'Université Cheikh Anta Diop de Dakar au Sénégal.

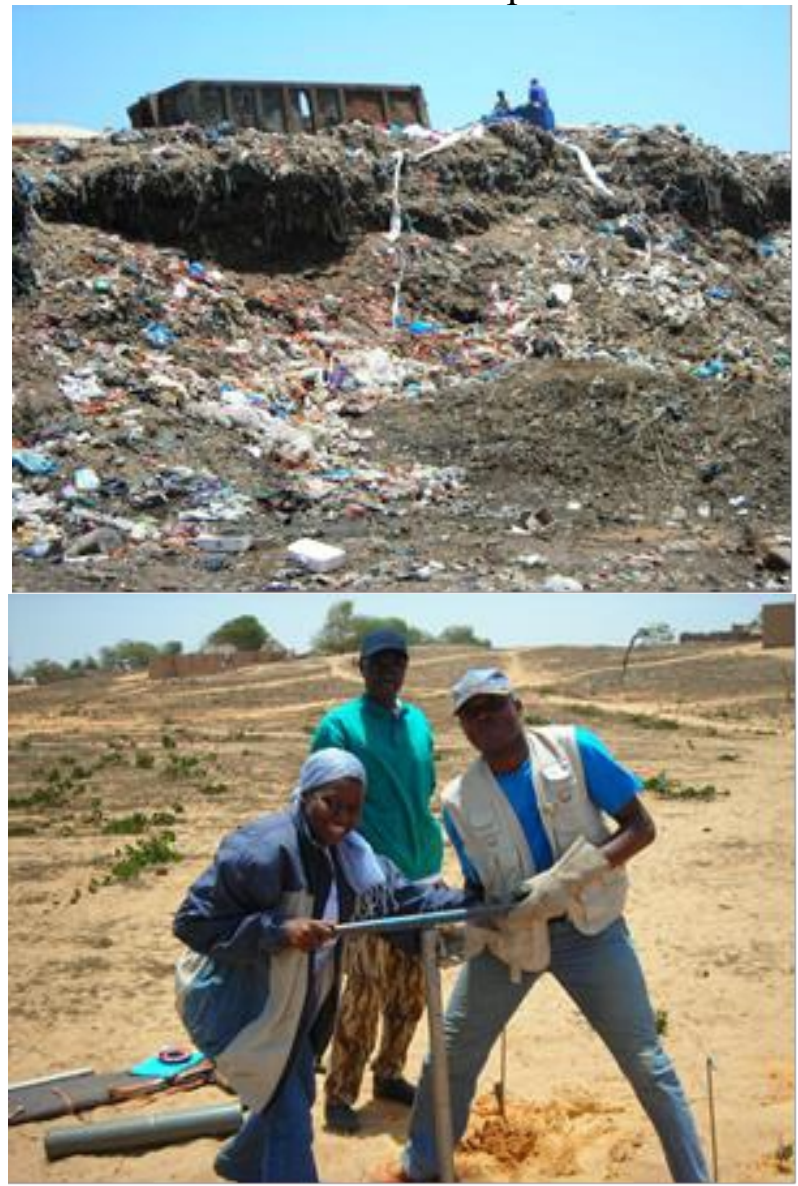

Figure 4. Décharge publique et prélèvement des échantillons de sols à la tarière manuelle.

La position géographique des points d'eau échantillonnés (puits traditionnels et piézomètres d'observation) et des profils des sols de la zone 
non saturée de l'aquifère des sables quaternaires réalisés, est reportée sur la Figure 5.
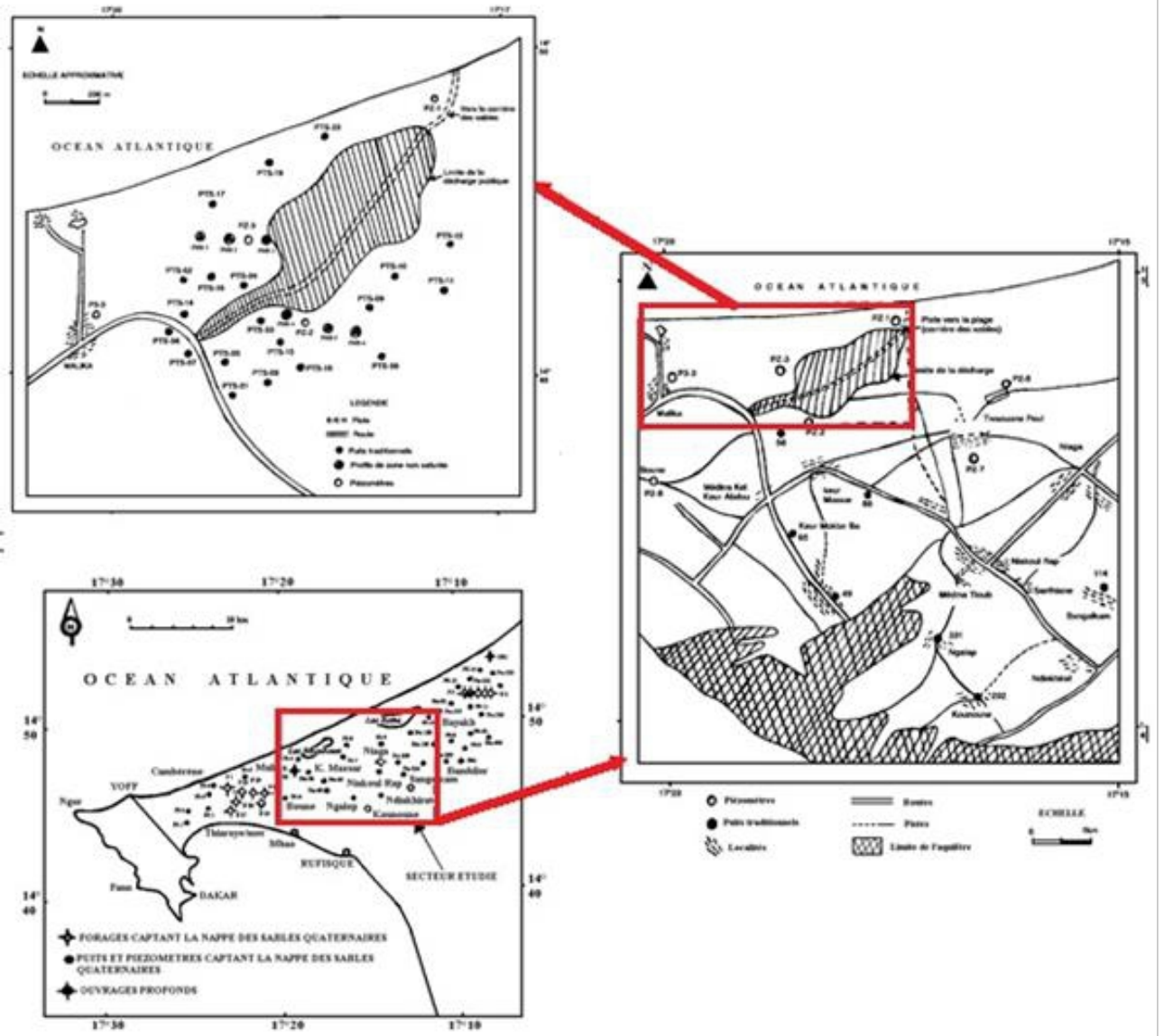

Figure 5. Localisation des ouvrages échantillonnés (Essouli, 2005).

Au laboratoire, les opérations suivantes ont été effectuées sur 10 à $50 \mathrm{~g}$ de sédiment:

- détermination de l'humidité pondérale des sols ;

- extraction des sels solubles par lixiviation des sols;

- analyses chimiques des éléments majeurs des eaux souterraines ;

- analyses chimiques des éléments majeurs sur les extraits aqueux des sols.

La teneur en eau pondérale des échantillons de sols a été déterminée par la méthode gravimétrique classique. Elle consiste à faire sécher un poids de sol connu pendant 24 heures à $150^{\circ} \mathrm{C}$. Dans le cadre de ce travail $20 \mathrm{~g}$ de sol ont été prélevés et séchés à l'étuve. Après le séchage, l'échantillon a été pesé à l'aide d'une balance de précision. 


\section{$\omega(\%)=\frac{\text { Poids initial de sol }- \text { Poids de sol sec }}{\text { Poids de sol sec }} * 100$ \\ Poids de sol sec}

La lixiviation est la méthode la plus couramment employée pour l'extraction des sels solubles de l'échantillon du sol. La technique consiste à mettre $50 \mathrm{~g}$ de sol humide dans un volume d'eau déminéralisée $(100 \mathrm{~mL})$ et à agiter pendant un temps jusqu'à l'obtention d'un bon mélange indiqué par la stabilité de la conductivité électrique de la solution.

Le mélange est filtré sur membrane millipores $(0,20 \mu \mathrm{m})$. Les teneurs chimiques obtenues sur le filtrat sont corrigées par rapport à l'humidité pondérale de l'échantillon $(\square)$ en considérant le facteur de dilution (df) :

$$
d_{f}=\frac{\frac{100}{50 * \omega(\%)}}{100 * \omega(\%)}+1
$$

La concentration $\mathrm{C}_{\mathrm{i}}$ de la solution initiale du sol s'exprime par : $C_{i}=C_{\text {filtrat }} \cdot d_{f}$.

Au laboratoire, les analyses chimiques des ions chlorures $\left(\mathrm{Cl}^{-}\right)$, sulfates $\left(\mathrm{SO}_{4}{ }^{2-}\right)$, nitrates $\left(\mathrm{NO}_{3}^{-}\right)$, sodium $\left(\mathrm{Na}^{+}\right)$, magnésium $\left(\mathrm{Mg}^{2+}\right)$ et calcium $\left(\mathrm{Ca}^{2+}\right)$ ont été effectuées par chromatographie ionique au Dionex 100S, celles des ions carbonates $\left(\mathrm{CO}_{3}{ }^{2-}\right)$ et bicarbonates $\left(\mathrm{HCO}_{3}{ }^{-}\right)$par titrimétrie et celles des ions phosphates $\left(\mathrm{PO}_{4}{ }^{3-}\right)$ par chromatographie à la flamme sur les eaux extraites des sols par lixiviation et les eaux souterraines.

Un contrôle systématique des erreurs relatives $(|\varepsilon|)$ sur les balances ioniques des résultats des analyses chimiques a été fait pour l'ensemble des points d'eau analysés au laboratoire. Les erreurs sont inférieures ou égales à 5 $\%$, ce qui témoigne d'une bonne qualité des analyses, et peuvent être traitées sans restriction à l'aide des logiciels de traitement des données hydrochimiques comme le diagramme développé par le laboratoire d'hydrogéologie de l'Université d'Avignon et Microsoft Excel.

\section{Résultats et Interprétations}

\subsection{Caractéristiques générales des eaux souterraines}

Dans le secteur de Thiaroye/lac Mbeubeuss, les valeurs de la conductivité électrique des eaux souterraines sont très hétérogènes (Tableau 1). Elles sont comprises entre $215 \mu \mathrm{S} / \mathrm{cm}$ (P3-3) et $4720 \mu \mathrm{S} / \mathrm{cm}$ (Pts-03).

La majorité des points d'eau échantillonnés ont des valeurs de la conductivité électrique qui sont inférieures à $2000 \mu \mathrm{S} / \mathrm{cm}$ (Norme OMS, 2004) 
et constituerait la véritable valeur de la conductivité électrique de la nappe des sables quaternaires (bruit de fond). Une telle valeur est raisonnable avec l'approche de l'océan et surtout pour une matrice aquifère constituée essentiellement de matériaux sableux. Cependant, une minorité des points d'eau situés aux alentours de la décharge publique ont des valeurs de la conductivité électrique qui sont supérieures à $2000 \mu \mathrm{S} / \mathrm{cm}$ (Norme OMS, 2004). Celles-ci démontrent $t$ une contamination des eaux souterraines par la décharge publique.

Concernant la qualité des eaux souterraines, l'aquifère des sables quaternaires dans le secteur de Thiaroye/lac Mbeubeuss présente une potabilité permanente entre les qualités bonne, passable, médiocre et mauvaise. Les paramètres chimiques analysés ont des teneurs maximales audessus des concentrations maximales admissibles fixées pour l'eau de boisson par l'Organisation Mondiale de la Santé (OMS, 2004). Les teneurs en nitrates élevées sont mesurées au niveau de certains puits villageois captant la nappe des sables quaternaires aux abords de la décharge publique et dans les localités à forte population. Ces fortes teneurs en nitrates dans le secteur du lac Mbeubeuss inhabité seraient probablement dues au lessivage par les eaux de pluies, des produits issus de la décomposition des ordures ménagères mises en décharge.

Tableau 1. Statistiques descriptives des paramètres chimiques des eaux souterraines $(\mathrm{mg} / \mathrm{L})$.

\begin{tabular}{|c|c|c|c|c|c|}
\hline $\begin{array}{c}\text { Paramètres } \\
\text { chimiques }\end{array}$ & Minimum & Maximum & Ecart-type & $\begin{array}{c}\text { Coefficient de } \\
\text { variation } \\
(\mathbf{C V} \text { en \%) }\end{array}$ & $\begin{array}{c}\text { Normes } \\
\text { OMS (2004) }\end{array}$ \\
\hline $\mathrm{CE}(\mu \mathrm{S} / \mathrm{cm})$ & 215 & 4720 & 95,87 & 92,49 & 2000 \\
\hline $\mathrm{Na}^{+}$ & 26 & 603,80 & 112,48 & 116,91 & 100 \\
\hline $\mathrm{K}^{+}$ & 2,37 & 35,55 & 8,22 & 90,03 & 12 \\
\hline $\mathrm{Ca}^{2+}$ & 15 & 225 & 61,37 & 83,74 & 100 \\
\hline $\mathrm{Mg}^{2+}$ & 1,35 & 104,44 & 22,82 & 111,43 & 50 \\
\hline $\mathrm{Cl}^{-}$ & 33,33 & 963,94 & 204,37 & 111,54 & 200 \\
\hline $\mathrm{SO}_{4}{ }^{2-}$ & 4,85 & 966,64 & 213,22 & 174,80 & 250 \\
\hline $\mathrm{HCO}_{3}{ }^{-}$ & 0 & 250,1 & 67,19 & 70,81 & - \\
\hline $\mathrm{NO}_{3}{ }^{-}$ & 0 & 221,42 & 57,73 & 83,74 & 50 \\
\hline $\mathrm{PO}_{4}{ }^{3}$ & 0,12 & 3,47 & 0,70 & 94,59 & 2,20 \\
\hline
\end{tabular}

La corrélation entre la conductivité électrique et les éléments chimiques montre que la minéralisation des eaux souterraines est essentiellement liée aux chlorures, sulfates, phosphates, sodium, potassium, magnésium et calcium. La contribution des bicarbonates et nitrates est très négligeable par rapport à celle des autres éléments chimiques et n'ont pas de rôle déterminant dans la minéralisation des eaux (Tableau 2). 
Tableau 2. Matrice de corrélation de l'aquifère des sables quaternaires.

\begin{tabular}{|c|c|c|c|c|c|c|c|c|c|c|}
\hline & $\mathrm{CE}$ & $\mathrm{HCO}_{3}^{-}$ & $\mathrm{Cl}^{-}$ & $\mathrm{SO}_{4}{ }^{2-}$ & $\mathrm{NO}_{3}{ }^{-}$ & $\mathrm{PO}_{4}{ }^{3-}$ & $\mathrm{Na}^{+}$ & $\mathbf{K}^{+}$ & $\mathrm{Mg}^{2+}$ & $\mathrm{Ca}^{2+}$ \\
\hline CE & 1 & & & & & & & & & \\
\hline $\mathrm{HCO}_{3}{ }^{-}$ & 0,10 & 1 & & & & & & & & \\
\hline $\mathrm{Cl}^{-}$ & 0,97 & $-0,15$ & 1 & & & & & & & \\
\hline $\mathrm{SO}_{4}{ }^{2-}$ & 0,92 & $-0,26$ & 0,85 & 1 & & & & & & \\
\hline $\mathrm{NO}_{3}{ }^{-}$ & 0,16 & $-0,22$ & 0,23 & $-0,07$ & 1 & & & & & \\
\hline $\mathrm{PO}_{4}{ }^{3-}$ & 0,77 & $-0,11$ & 0,76 & 0,74 & $-0,09$ & 1 & & & & \\
\hline $\mathrm{Na}^{+}$ & 0,94 & $-0,23$ & 0,97 & 0,87 & 0,19 & 0,75 & 1 & & & \\
\hline $\mathbf{K}^{+}$ & 0,88 & $-0,20$ & $\mathbf{0 , 8 7}$ & 0,83 & 0,27 & 0,63 & 0,84 & 1 & & \\
\hline $\mathrm{Mg}^{2+}$ & 0,97 & $-0,23$ & 0,97 & 0,88 & 0,24 & 0,75 & 0,94 & 0,89 & 1 & \\
\hline $\mathrm{Ca}^{2+}$ & 0,85 & $-0,20$ & 0,75 & 0,74 & 0,09 & 0,56 & 0,65 & 0,66 & 0,75 & 1 \\
\hline
\end{tabular}

Les données des analyses chimiques de juillet 2002 sont reportées sur les diagrammes triangulaires de Piper pour déterminer les familles et faciès géochimiques des eaux de l'aquifère des sables quaternaires (Figure 6).

L'interprétation des résultats du diagramme de Piper montre que les eaux souterraines sont dominées par les familles chimiques chlorurées sulfatées calciques et magnésiennes et chlorurées sulfatées sodiques et potassiques. Les faciès chimiques sont chloruré sodique et potassique et chloruré calcique.

Dans les détails, l'évolution chimique des eaux souterraines se présente comme suit : dans le diagramme triangulaire des anions, nous distinguons trois groupes de points : le premier proche du pôle chloruré $(72 \%)$, le deuxième à évolution vers le pôle sulfaté $(8 \%)$ et le troisième vers la zone centrale mixte $(20 \%)$. Dans le diagramme triangulaire des cations, il y a aussi trois groupes : le premier à évolution vers le pôle sodique et potassique (36\%), le deuxième vers le pôle calcique $(8 \%)$ et le troisième vers la zone centrale mixte de non prédominance des différents cations $(56 \%)$. 


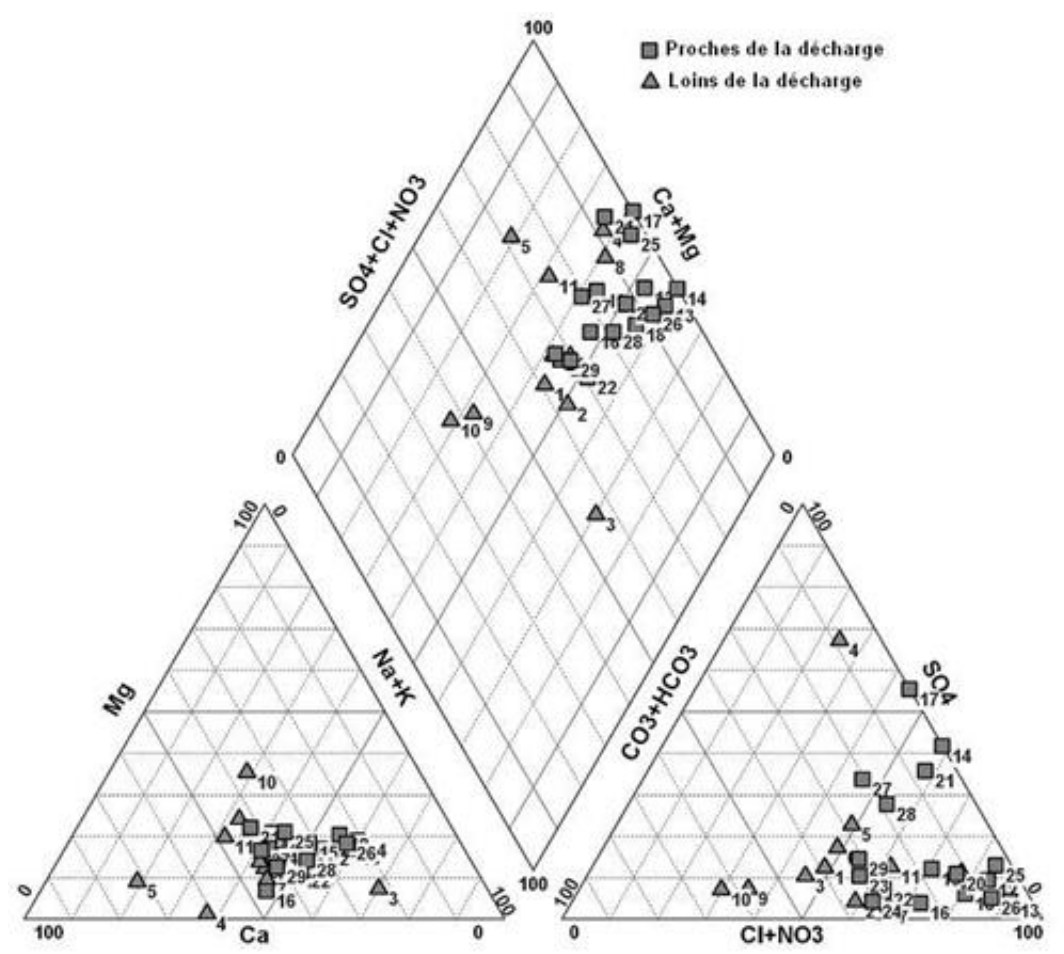

Figure 6. Diagrammes triangulaires de Piper pour l'aquifère des sables quaternaires.

\section{2- Origine et processus de minéralisation des eaux}

Le faciès chimique naturel des eaux souterraines répond à un déterminisme en partie dû à la nature lithologique des aquifères et des terrains superficiels traversés par l'eau. Les descriptions lithologiques de l'aquifère montrent une matrice quasi-exclusivement sableuse. Dans ce contexte géologique, l'origine de la minéralisation des eaux dans cet aquifère du secteur de Thiaroye/lac Mbeubeuss n'est pas seulement liée à la lithologie de l'aquifère. Elle peut être due probablement aux apports météoriques (pluie et évaporation), à l'acquisition des éléments chimiques par dissolution ou altération des minéraux argileux présent dans le réservoir et aux apports de surface par percolation dans la zone non saturée au cours de l'infiltration des eaux de pluie (lessivage du sol et lixiviation de la matière organique provenant des ordures ménagères) et des processus d'échange de base entre les eaux souterraines et la matrice de l'aquifère.

En tenant compte de la concentration des ions majeurs des eaux souterraines, les concentrations observées en juillet 2002 sont très élevées pour une matrice aquifère constituée de matériaux sableux à sablo-argileux et alimentée essentiellement par les eaux de pluie (Tableau 3). Ces résultats impliquent une source extérieure de minéralisation de la nappe des sables quaternaires par les eaux de pluies au cours de leur infiltration, d'où l'intérêt 
d'étudier la variation des teneurs en éléments majeurs des lixiviats des sols de la zone non saturée de l'aquifère, les indices de saturation vis-à-vis des différentes phases minérales et la relation entre les concentrations des différents éléments majeurs et ces chlorures.

Tableau 3. Composition chimique moyenne des eaux de pluies (Travi et al., 1987) et de l'aquifère des sables quaternaires à Mbeubeuss en juillet 2002 .

\begin{tabular}{|c|c|c|c|}
\hline Paramètres chimiques & $\begin{array}{c}\text { Stations } \\
\text { pluviométriques }\end{array}$ & $\begin{array}{c}\text { dans les eaux de } \\
\text { pluies }(\mathbf{m g} / \mathbf{L})\end{array}$ & $\begin{array}{c}\text { dans la nappe de Thiaroye } \\
\text { au site du lac Mbeubeuss en } \\
\text { juillet 2002 (mg/L) }\end{array}$ \\
\hline Sodium $\left(\mathrm{Na}^{+}\right)$ & Dakar & 3,36 & 97,37 \\
\hline Potassium $\left(\mathrm{K}^{+}\right)$ & Dakar & 0,57 & 9,19 \\
\hline Calcium $\left(\mathrm{Ca}^{2+}\right)$ & Dakar & 1,7 & 69,89 \\
\hline Magnésium $\left(\mathrm{Mg}^{2+}\right)$ & Dakar & 0,39 & 18,90 \\
\hline Sulfates $\left(\mathrm{SO}_{4}^{2-}\right)$ & Dakar & 2,42 & 65,86 \\
\hline Nitrates $\left(\mathrm{NO}_{3}^{-}\right)$ & Dakar & 0,07 & 77,80 \\
\hline Bicarbonates $\left(\mathrm{HCO}_{3}^{-}\right)$ & Saint Louis & 2,21 & 179,15 \\
\hline Chlorures $\left(\mathrm{Cl}^{-}\right)$ & Saint Louis & 2,26 & \\
\hline
\end{tabular}

\subsubsection{Influence de la zone non saturée sur la minéralisation des eaux souterraines}

Six profils de moins de $3 \mathrm{~m}$ de profondeur de la surface du sol jusqu'à la nappe ont été réalisés dans la zone non saturée de l'aquifère des sables quaternaires en juillet 2002 en fin de saison sèche, moment où les phénomènes évaporatoires sont intenses. Ils sont situés sur l'axe des piézomètres (PZ-2 et PZ-3) de surveillance de la nappe des sables quaternaires au site du lac Mbeubeuss : de la décharge publique vers le centre du lac Mbeubeuss (PMB1, PMB-2 et PMB-3) et de la décharge publique vers la bordure du lac Mbeubeuss (PMB-4, PMB-5 et PMB-6) suivant un trajet globalement ouest/est. Ces profils de zone non saturée ont été réalisés dans le but d'étudier l'évolution verticale et latérale des teneurs en éléments majeurs en fonction de la profondeur de part et d'autre de la décharge publique en s'éloignant de cette dernière. Cette étude de la zone non saturée est entreprise avec l'objectif de mettre en évidence l'implication de la décharge publique dans la minéralisation de la nappe des sables quaternaires dans le secteur de Thiaroye/lac Mbeubeuss.

Les variations des teneurs en $\mathrm{Cl}^{-}, \mathrm{SO}_{4}{ }^{2-}, \mathrm{NO}_{3}{ }^{-}, \mathrm{PO}_{4}{ }^{3-}, \mathrm{Na}^{+}, \mathrm{K}^{+}, \mathrm{Ca}^{2+}$ et $\mathrm{Mg}^{2+}$ des extraits aqueux des sols de l'aquifère en fonction de la profondeur sont données par les Figures 7 et 8. On peut alors distinguer suivant la verticale :

une zone superficielle de forte concentration en $\mathrm{Cl}^{-}, \mathrm{SO}_{4}{ }^{2-}, \mathrm{NO}_{3}{ }^{-}, \mathrm{PO}_{4}{ }^{3-}$ $, \mathrm{Na}^{+}, \mathrm{K}^{+}, \mathrm{Ca}^{2+}$ et $\mathrm{Mg}^{2+}$ à cause d'une accumulation due à la reprise évaporatoire. Le front d'évaporation varie d'un profil à l'autre suivant l'état hydrique. Cette partie du profil correspond à la zone où le transit 
en phase vapeur est le mécanisme dominant du mouvement ascendant ou descendant de l'eau et des solutés du sol (Barnes \& Allison, 1983);

- $\quad$ une zone profonde de faible concentration en $\mathrm{Cl}^{-}, \mathrm{SO}_{4}{ }^{2-}, \mathrm{NO}_{3}{ }^{-}, \mathrm{PO}_{4}{ }^{3-}$, $\mathrm{Na}^{+}, \mathrm{K}^{+}, \mathrm{Ca}^{2+}$ et $\mathrm{Mg}^{2+}$ due à l'augmentation de l'humidité pondérale qui entraîne des phénomènes de dilution. Dans cette partie domine le transit liquide (Zouari et $a l .$, 1984). Sur les profils, les maxima d'enrichissement qui définissent la zone de transition entre la remontée capillaire et le flux d'évaporation se situent à des profondeurs d'environ 62,5 cm sur PMB-1 et PMB-6 et 12,5 cm sur PMB-4 et PMB-6. En plus, suivant l'axe d'orientation ouest/est des profils de zone non saturée, de part et d'autre de la décharge publique, les teneurs en $\mathrm{Cl}^{-}, \mathrm{SO}_{4}{ }^{2-}, \mathrm{NO}_{3}{ }^{-}, \mathrm{PO}_{4}{ }^{3-}, \mathrm{Na}^{+}, \mathrm{K}^{+}, \mathrm{Ca}^{2+}$ et $\mathrm{Mg}^{2+}$ sont plus élevées sur les profils (PMB-1 et PMB-4) plus proches de la décharge.

Pour mettre en évidence l'impact de la décharge publique sur la minéralisation des eaux souterraines aux environs du lac Mbeubeuss, les résultats des analyses chimiques des lixiviats des sols du site du lac Mbeubeuss ont été comparés aux résultats des études antérieures menées sur d'autres sites de la région de Dakar (Gaye, 1990 ; Tandia, 2000). Cette comparaison fait ressortir les constatations suivantes :

l'allure des profils de la zone non saturée réalisés à Mbeubeuss est comparable à celle obtenue par Gaye (1990) dans la zone de Niague et par Tandia (2000) dans le secteur de Yeumbeul. Celle-ci est liée à une accumulation des éléments chimiques majeurs dans les horizons superficiels sous l'effet de l'évaporation. Ces observations sont en accord avec celles de Edmunds et Walton (1980) et Gouvea Da Silva Rosa (1980); 


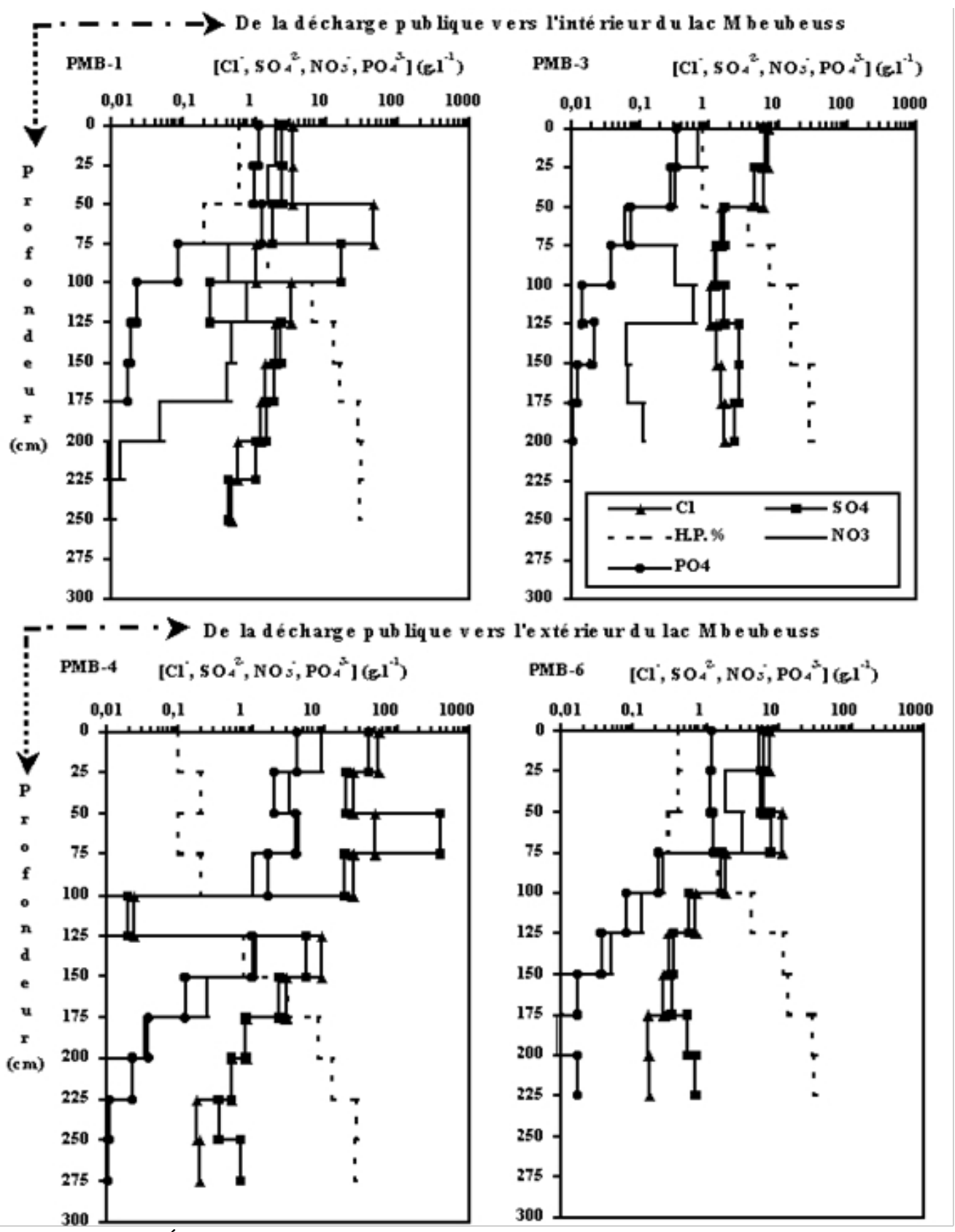

Figure 7. Évolution des teneurs en éléments chimiques dans la zone non saturée. 

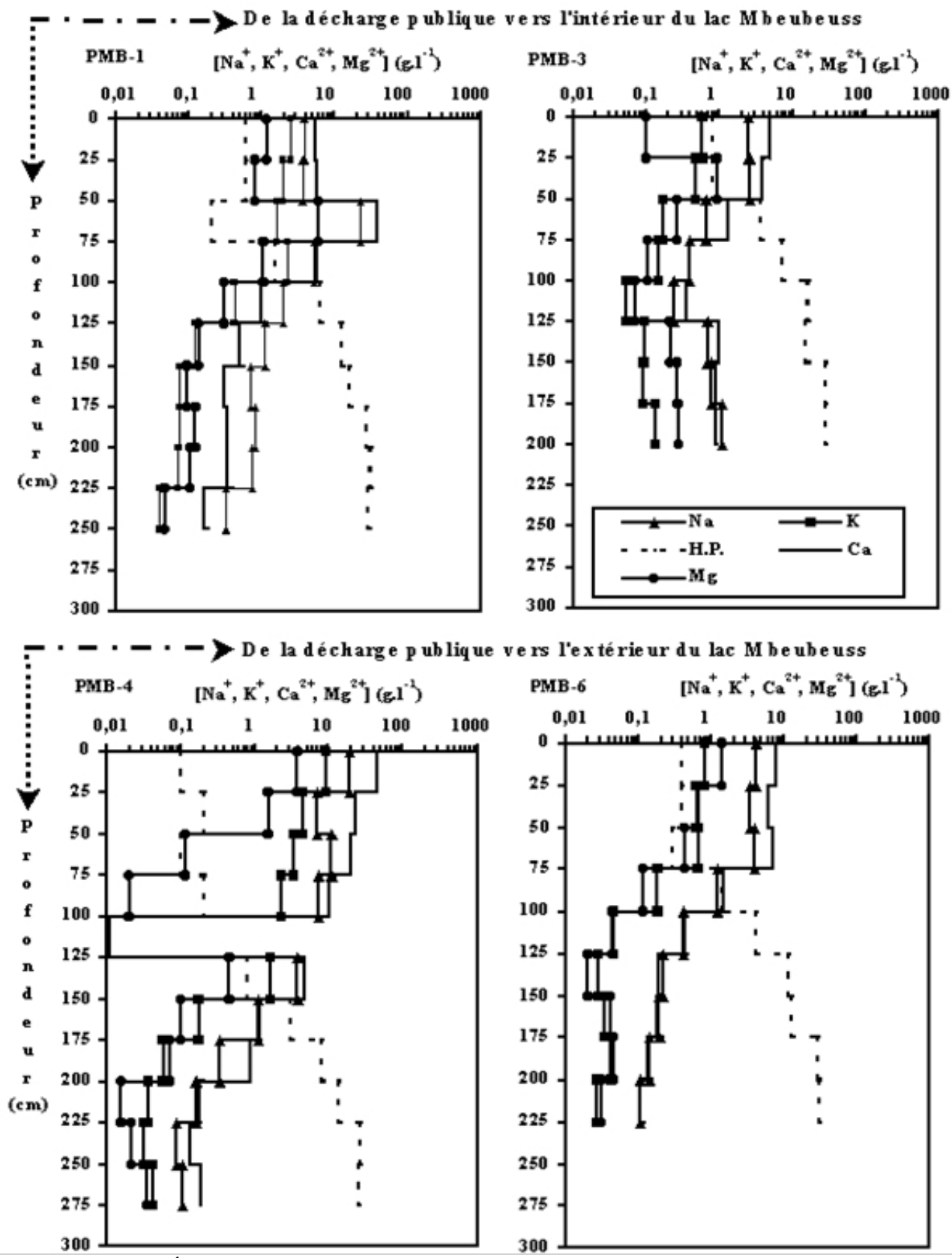

Figure 8. Évolution des teneurs en éléments chimiques dans la zone non saturée.

- les accumulations dans les horizons superficiels des sols sont plus importantes sur les profils réalisés à proximité de la décharge publique du site du lac Mbeubeuss (Tableau 4) que sur les profils qui ont été réalisés dans les secteurs de Niague par Gaye (1990) et de Yeumbeul par Tandia (2000). Ainsi , plusieurs hypothèses sont émises pour 
expliquer les fortes teneurs en éléments chimiques dans la zone non saturée au site du lac Mbeubeuss, telle que la situation à proximité de la mer qui laisse supposer un apport considérable de sels par les aérosols et embruns marins.

Tableau 4. Concentrations moyennes en ions majeurs des lixiviats des sols de la zone non saturée aux abords de la décharge (juillet 2002)

\begin{tabular}{|l|c|c|c|c|c|c|}
\hline & PMB-1 & PMB-2 & PMB-3 & PMB-4 & PMB-5 & PMB-6 \\
\hline H.P. (\%) & 12,47 & 12,86 & 12,40 & 7,16 & 6,02 & 10,14 \\
\hline $\mathbf{C l}^{-}(\mathbf{g} / \mathbf{l})$ & 6,13 & 10,88 & 2,62 & 15,34 & 7,14 & 3,13 \\
\hline $\mathbf{S O}_{4}{ }^{2-}(\mathbf{g} / \mathbf{l})$ & 3,32 & 113,01 & 2,89 & 44,88 & 5,52 & 2,65 \\
\hline $\mathbf{N O}_{3}{ }^{-}(\mathbf{g} / \mathbf{l})$ & 1,140 & 3,134 & 0,281 & 1,742 & 1,243 & 1,197 \\
\hline $\mathbf{P O}_{4}{ }^{3-}(\mathbf{g} / \mathbf{l})$ & 0,358 & 0,493 & 0,096 & 1,183 & 0,871 & 0,434 \\
\hline $\mathbf{N a}^{+}(\mathbf{g} / \mathbf{l})$ & 4,11 & 4,27 & 1,10 & 4,54 & 3,52 & 1,55 \\
\hline $\mathbf{K}^{+}(\mathbf{g} / \mathbf{l})$ & 2,339 & 0,808 & 0,221 & 1,930 & 1,010 & 0,274 \\
\hline $\mathbf{C a}^{2+}(\mathbf{g} / \mathbf{l})$ & 5,81 & 28,98 & 1,71 & 9,52 & 19,56 & 2,57 \\
\hline $\mathbf{M g}^{2+}(\mathbf{g} / \mathbf{l})$ & 0,986 & 2,518 & 0,389 & 0,550 & 1,328 & 0,312 \\
\hline
\end{tabular}

L'augmentation des teneurs en solutés de la zone non saturée par le lessivage des produits de la décomposition des ordures ménagères par les eaux des pluies au cours de l'infiltration vers la nappe, se fait sous l'effet des phénomènes d'évaporation. Au site de Mbeubeuss, l'ancien lac salé asséché jouerait également un rôle important dans la minéralisation de solutions des sols par dissolution des anciens dépôts évaporitiques et par conséquent des eaux souterraines. Ainsi les principaux mécanismes contrôlant le transfert des polluants sont essentiellement les flux verticaux et horizontaux liés au lessivage d'ordures ménagères, les embruns marins et aérosols, sous l'action des eaux de pluie.

La présence de coquillages dans les formations argilo-sableuses de la zone non saturée entre 0,25 et $0,75 \mathrm{~m}$ de profondeur sur le profil PMB-2, témoigne un milieu marin ancien fermé. Dans des conditions où l'évaporation est excédentaire par rapport aux précipitations et aux apports d'eaux marines et continentales, tous les sels évaporitiques peuvent précipiter. C'est ce que l'on observe dans les lagunes et lacs côtiers évaporitiques actuels des pays chauds et secs (Chamley, 2000). Cette observation laisse penser que la minéralisation des eaux interstitielles des sols est influencée par la dissolution des coquillages calcaires et/ou par l'évaporation des eaux de mer anciennes piégées dans les formations argilo-sableuses de subsurface, ce qui favorise l'accumulation des sels dans la zone non saturée. Ceci pourrait expliquer les concentrations élevées en éléments chimiques majeurs sur le profil PMB-2 et supposerait que les minéralisations anciennes du lac Mbeubeuss, en plus de la décharge, contribuent à la contamination des eaux souterraines. Par ailleurs, la nature de l'aquifère dans cette zone est favorable aux transferts des polluants 
vers la nappe. C'est ainsi que les principaux mécanismes contrôlant ces transferts résulteraient essentiellement des flux verticaux et horizontaux liés au lessivage des déchets d'ordures ménagères, des embruns marins et des aérosols sous l'action des eaux de pluie.

L'étude des variations de concentrations en éléments chimiques majeurs dans la zone non saturée de l'aquifère, permet de préciser les différentes sources susceptibles de contribuer à l'augmentation de la minéralisation des lixiviats. En effet, la proximité de la mer laisse supposer un apport considérable des sels par les aérosols et embruns marins. A cet apport s'ajoute l'influence à la fois des dépôts anciens du lac Mbeubeuss asséché et le lessivage de la matière organique provenant de la décomposition des ordures ménagères de la décharge publique par les eaux de pluies. De plus, étant la nappe à moins de $3 \mathrm{~m}$ de profondeur dans le secteur d'étude, les teneurs en éléments chimiques des lixiviats des sols pourraient être influencées par la reprise évaporatoire qui provoque l'augmentation de leurs concentrations.

\subsection{2. Étude des éléments majeurs des eaux souterraines}

Pour discuter de l'origine et des processus de minéralisation des eaux souterraines dans le secteur de Thiaroye/lac Mbeubeuss, les indices de saturation (IS) vis-à-vis des phases minérales présentes (anhydrite $\left(\mathrm{CaSO}_{4}\right)$, aragonite $\left(\mathrm{CaCO}_{3}\right)$, calcite $\left(\mathrm{CaCO}_{3}\right)$, dolomite $\left(\mathrm{CaMg}\left(\mathrm{CO}_{3}\right)_{2}\right)$, magnésite $\left(\mathrm{MgCO}_{3}\right)$ et gypse $\left(\mathrm{CaSO}_{4}, \mathrm{H}_{2} \mathrm{O}\right)$, ont été calculés à partir du programme WATEQ (Truesdel \& Jones, 1974 ; Plummer et al., 1976).

La relation entre les indices de saturation et la minéralisation totale "TDS" (Dindane et al., 2003) montre que les eaux souterraines dans le secteur de Thiaroye/lac Mbeubeuss sont sous-saturées vis- à -vis de l'anhydrite et du gypse et sous saturées à sursaturées vis- à- vis de l'aragonite, de la calcite, de la dolomite et de la magnésite. L'état de sous-saturation suppose, a priori, une possibilité de dissolution des phases minérales et l'état de sursaturation suppose, a priori, une possibilité de précipitation des phases minérales (Figure 9). 


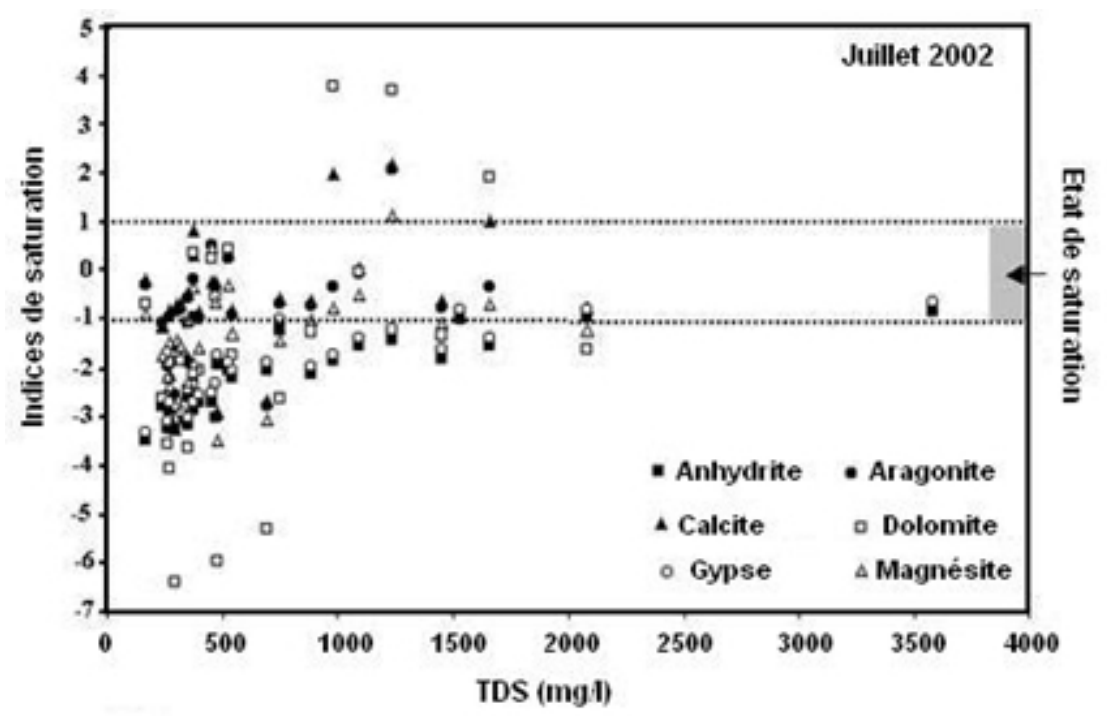

Figure 9. Évolution des indices de saturation en fonction du TDS.

La Figure 10 montre la relation entre les chlorures et les éléments majeurs $\left(\mathrm{SO}_{4}{ }^{2-}, \mathrm{HCO}_{3}{ }^{-}, \mathrm{Ca}^{2+}, \mathrm{Mg}^{2+}, \mathrm{Na}^{+}\right.$et $\left.\mathrm{K}^{+}\right)$des points d'eaux prélevés dans le secteur de Thiaroye/lac Mbeubeuss. La disposition des différents points par rapport à la droite de mélange eau douce-eau salée (eau de pluie-eau de mer) peuvent être de grande utilité pour identifier d'autres phénomènes qui sont annexés au processus de mélange. Les eaux souterraines des différents points d'eau montrent en général un enrichissement en $\mathrm{Ca}^{2+}, \mathrm{HCO}_{3}{ }^{-}$et légèrement en $\mathrm{SO}_{4}{ }^{2-}$ et un appauvrissement en $\mathrm{Na}^{+}$et $\mathrm{K}^{+}$, tandis que pour le $\mathrm{Mg}^{2+}$ nous avons une dispersion des points autour de la droite de mélange. Les réactions majeures reconnues comme responsables d'enrichissements ou d'appauvrissements des éléments majeurs et de traces dans les eaux souterraines sont (Fidelibus et al., 1996) : (a) échange $\mathrm{Ca}^{2+} \Leftrightarrow \mathrm{Mg}^{2+}$ dû à l'interaction eau-roches, (b) $\mathrm{Na}^{+} \Leftrightarrow \mathrm{Ca}^{2+}$ ou $\mathrm{Na}^{+} \Leftrightarrow \mathrm{Mg}^{2+}$ par des échanges de base, (c) réduction des sulfates.

Les diagrammes des évolutions des teneurs en sulfates et en bicarbonates des eaux souterraines par rapport aux ions chlorures de l'eau de pluie et de l'eau de mer montrent un nuage de points situés au-dessus de la droite de mélange théorique eau de pluie/eau de mer. 

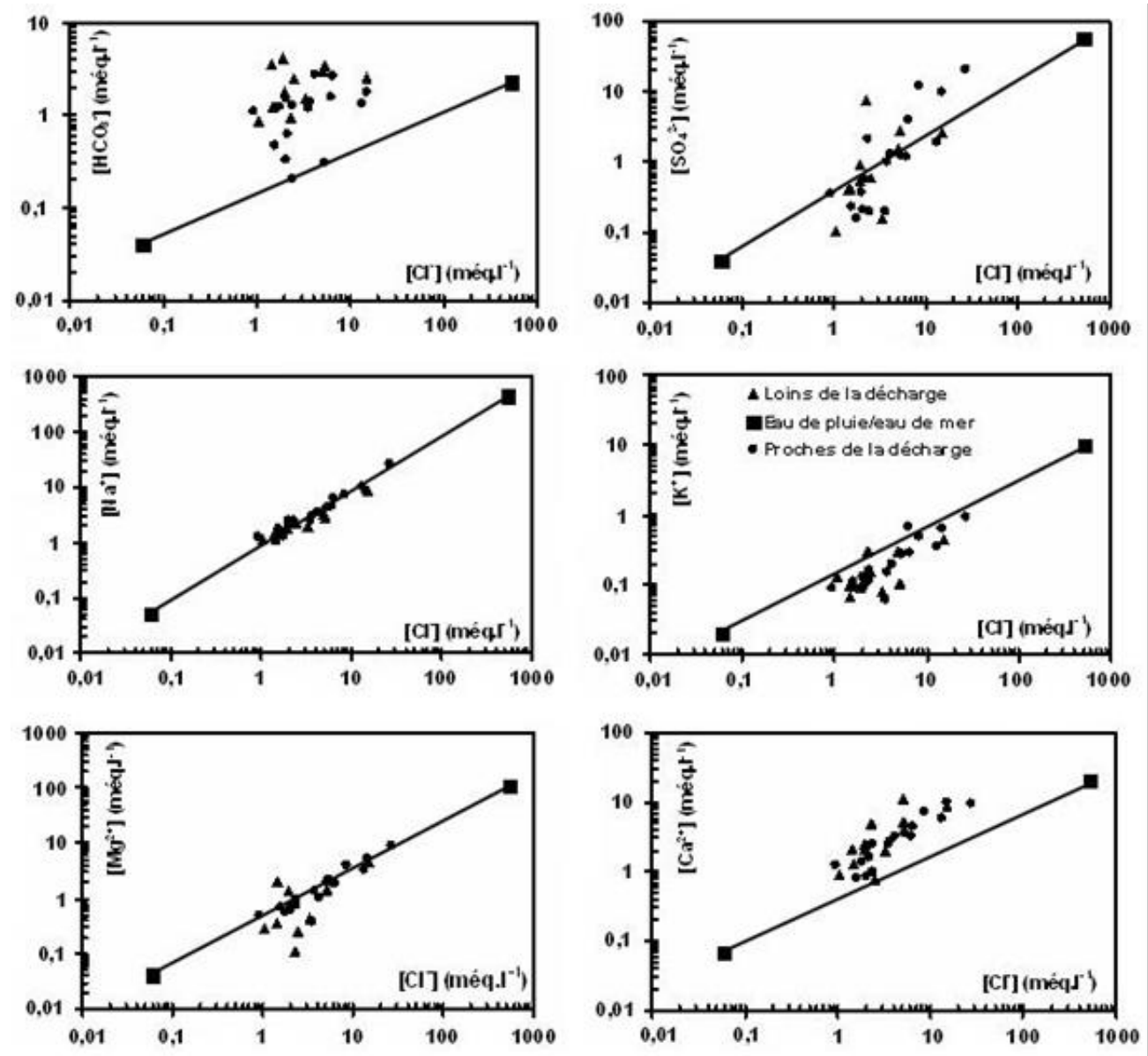

Figure 10. Relation entre les éléments chimiques majeurs et l'ion chlorure.

En ce qui concerne les bicarbonates, la position des points montre une tendance à un décalage vertical par rapport à la droite théorique et à l'étalement. Ces eaux ont des concentrations en ions bicarbonates supérieures à celles de l'eau de mélange théorique entre l'eau de pluie et l'eau de mer. Les matières organiques provenant de la décomposition des ordures ménagères de la décharge publique du lac Mbeubeuss, contribuent également à la production des bicarbonates. Ainsi, dans le secteur de Thiaroye, en plus de l'influence très probable du substratum carbonaté de l'aquifère, il faudrait ajouter certainement l'influence de la diffusion du gaz carbonique $\left(\mathrm{CO}_{2}\right)$ sur la production de bicarbonates.

Pour les sulfates, la majorité des points d'eaux sont situés au-dessus de la courbe de dilution et qui présentent des rapports molaires $\mathrm{SO}_{4}{ }^{2-} / \mathrm{Cl}^{-}$ supérieurs à celui de l'eau de mer. Cet enrichissement peut résulter de la zone non saturée de l'aquifère des sables quaternaires par décomposition et lessivage des déchets domestiques lors de la percolation de l'eau de pluie vers 
la nappe et du transport des aérosols marins qui contiennent des teneurs en sulfates élevées par rapport aux chlorures. En effet, les déchets domestiques produisent des quantités importantes de sulfates et les eaux de pluies des régions côtières du Sénégal montrent des rapports $\mathrm{SO}_{4}{ }^{2-} / \mathrm{Cl}^{-}$relativement faibles mais qui demeurent clairement élevées par rapport à celui de l'eau de mer (Travi et al., 1987).

Pour le sodium, on remarque que le nuage de points est au-dessus de la droite de mélange théorique entre l'eau de pluie et l'eau de mer. Le comportement des points d'eaux du voisinage du pôle de l'eau de pluie au voisinage du pôle de l'eau de mer met en évidence une succession de phénomènes de concentration et de dilution.

Pour le potassium, les échantillons d'eaux sont situés de part et d'autre de la droite théorique de mélange de l'eau de pluie et de l'eau de mer. Les échantillons situés au-dessus de la droite, ont des concentrations en potassium supérieures à celles de l'eau de mélange entre l'eau de pluie et l'eau de mer. Dans le secteur de Thiaroye/lac Mbeubeuss, on pourrait lier 1'enrichissement en ions potassium à un apport dû au lessivage des ordures ménagères de la décharge publique au cours de l'infiltration des eaux de pluie vers la nappe. L'étude de la zone non saturée dans l'environnement immédiat de la décharge publique a montré une concentration dans les horizons superficiels des teneurs élevées en ions potassium. On peut aussi ajouter un apport dû à la dissolution des minéraux argileux contenus dans les sédiments sableux.

La relation entre les teneurs en ions calcium des eaux souterraines et les teneurs en ions chlorures de l'eau de pluie et de l'eau de mer, montre un nuage des points d'eau situés au-dessus de la droite de mélange théorique. La position des points montre une tendance à un décalage vertical par rapport à la droite et à un étalement. Le décalage et le regroupement des points audessus de la droite de dilution suggèrent un apport supplémentaire par rapport aux teneurs de l'eau de mélange théorique et l'étalement des points qui se rapprochent du pôle de l'eau de mer met en évidence des phénomènes de concentration par évaporation.

Pour le magnésium, les points d'eau sont situés de part et d'autre de la droite théorique de dilution. Les points situés au-dessus de la droite ont des concentrations en magnésium supérieures à celles de l'eau de mélange entre l'eau de pluie et l'eau de mer. Dans la zone de Thiaroye, on peut lier la minéralisation en ions magnésium à un apport dû au lessivage des ordures ménagères de la décharge publique au cours de la percolation vers la nappe des eaux de pluie. En effet, l'étude de la zone non saturée aux abords immédiats de la décharge a montré des teneurs en ions magnésium très élevées de la surface vers la nappe et il suffit d'un événement pluvieux pouvant provoquer l'infiltration des eaux pour entraîner les sels vers la nappe. Quant 
aux points d'eaux situés sous la droite, ils représentent des eaux ayant de plus faibles concentrations en ions magnésium.

Les échanges de base qui caractérisent les différentes formations aquifères et les eaux souterraines sont mis en évidence par la relation entre $\left[\left(\mathrm{Ca}^{2+}+\mathrm{Mg}^{2+}\right)-\left(\mathrm{HCO}_{3}^{-}+\mathrm{SO}_{4}^{2-}\right)\right]$ et $\left[\left(\mathrm{Na}^{+}+\mathrm{K}^{+}\right)-\mathrm{Cl}^{-}\right]$, représentée par la Figure 11 (García et al., 2001).

Cette relation met l'accent uniquement sur les réactions pouvant exister entre les minéraux argileux et la solution, en se débarrassant des ions issus éventuellement d'autres réactions de dissolution des minéraux carbonatés et évaporitiques. Cette figure met en évidence les échanges de base avec les minéraux argileux qui affectent les eaux de la nappe des sables quaternaires dans le secteur de Thiaroye/lac Mbeubeuss pendant l'infiltration des eaux de pluies et durant leur séjour au sein de l'aquifère. Dans le cas d'absence de ces réactions, tous les points représentatifs des échantillons doivent se placer près du point d'origine (Mclean et $a l .$, 2000). Quatre zones sont énumérées à cette fin :

- les zones 1 et 2 , où la différence entre les ions $\left(\mathrm{Ca}^{2+}\right.$ et $\left.\mathrm{Mg}^{2+}\right)$ et les ions $\left(\mathrm{HCO}_{3}{ }^{-}\right.$et $\left.\mathrm{SO}_{4}{ }^{2-}\right)$ est positive, montre que dans ces deux zones c'est le processus d'échange inverse de bases qui domine. Les rapports négatifs sont dus à la rétention des alcalins au niveau des argiles et les rapports positifs à un apport d'ions $\mathrm{Na}^{+}$et $\mathrm{K}^{+}$. Cet apport reste cependant faible à cause des fortes perméabilités ;

- les zones 3 et 4 , où la différence entre les ions $\left(\mathrm{Ca}^{2+}\right.$ et $\left.\mathrm{Mg}^{2+}\right)$ et les ions $\left(\mathrm{HCO}_{3}{ }^{-}\right.$et $\left.\mathrm{SO}_{4}{ }^{2-}\right)$ est négative, indique que c'est le processus d'échange de base qui domine. Dans ces zones, on a une libération des ions alcalins $\left(\mathrm{Na}^{+}\right.$et $\left.\mathrm{K}^{+}\right)$et une rétention des alcalino-terreux $\left(\mathrm{Ca}^{2+}\right.$ et $\left.\mathrm{Mg}^{2+}\right)$ au niveau des argiles.

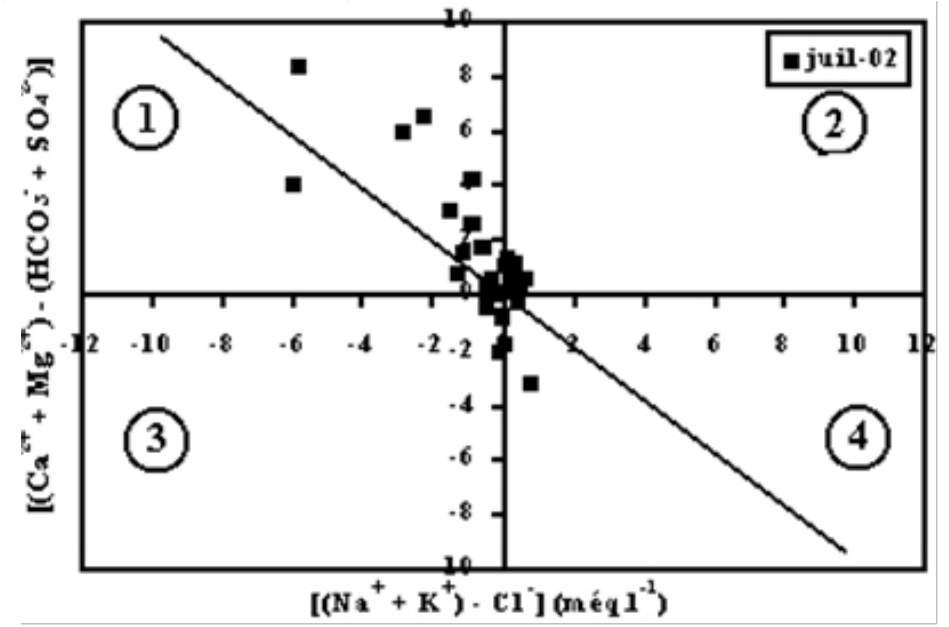

Figure 11. Diagramme binaire $\left[\left(\mathrm{Na}^{+}+\mathrm{K}^{+}\right)-\mathrm{Cl}^{-}\right]$vs $\left[\left(\mathrm{Ca}^{2+}+\mathrm{Mg}^{2+}\right)-\left(\mathrm{HCO}_{3}{ }^{-}+\mathrm{SO}_{4}{ }^{2-}\right)\right]$ des eaux souterraines. 
L'effet de la dissolution des minéraux évaporitiques et carbonates dans les formations aquifères et les eaux souterraines peut être mis en évidence par la relation entre $\left(\mathrm{Ca}^{2+}+\mathrm{Mg}^{2+}\right)$ et $\left(\mathrm{HCO}_{3}{ }^{-}+\mathrm{SO}_{4}{ }^{2-}\right)$. Cette relation, illustrée par la Figure 12, montre nettement que ce sont les points d'eau de l'aquifère des sables quaternaires dans le secteur de Thiaroye/lac Mbeubeuss placés sur ou proche de la droite de pente 1 dite la droite théorique de dissolution des minéraux argileux, ceux qui sont concernés par l'effet de la dissolution.

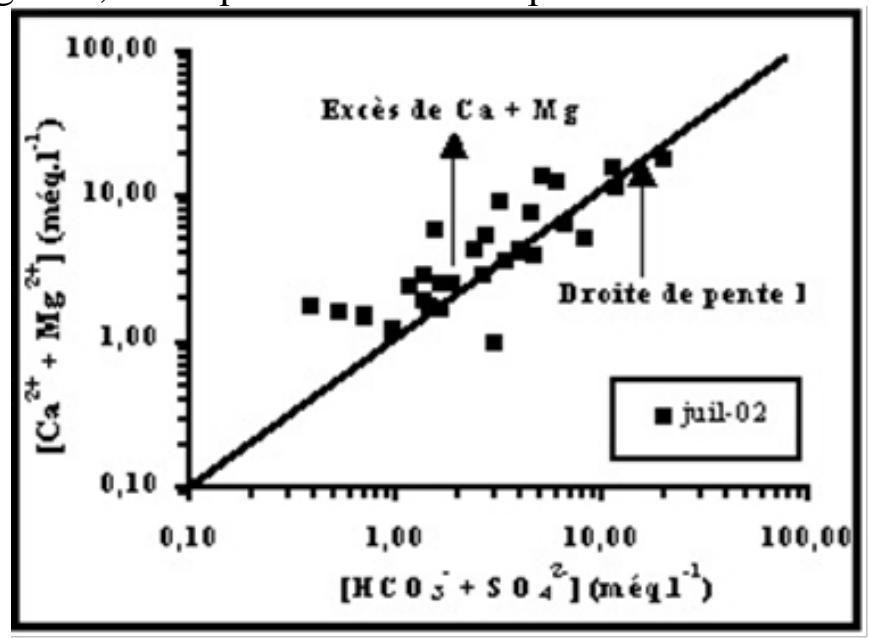

Figure 12. Diagramme binaire de dissolution $\left[\mathrm{HCO}_{3}{ }^{-}+\mathrm{SO}_{4}{ }^{2-}\right]$ vs $\left[\mathrm{Ca}^{2+}+\mathrm{Mg}^{2+}\right]$ des eaux souterraines.

\section{Conclusion}

L'aquifère des sables quaternaires dans le secteur de Thiaroye/lac Mbeubeuss présente une vulnérabilité à la pollution anthropique à cause de la faible profondeur de la nappe par rapport au sol et de la nature du réservoir constitué essentiellement de matériaux sableux très perméables facilitant le transfert rapide des polluants de la surface du sol vers la nappe.

L'étude de la zone non saturée et de la relation entre les éléments chimiques majeurs et l'ion chlorure a permis de préciser d'une part les sources de minéralisation des eaux souterraines dans le secteur de Thiaroye/lac Mbeubeuss et d'autre part les processus qui contrôlent la minéralisation des eaux souterraines. En effet, la situation à proximité de la mer laisse supposer un apport considérable de sels par les aérosols et embruns marins. A cet apport s'ajoute l'influence des anciens dépôts du lac Mbeubeuss asséché par précipitation et dissolution. Les activités anthropiques, les lixiviats provenant de la décomposition des ordures domestiques de la décharge publique ont des concentrations très importantes en ions majeurs. L'évaporation à la surface du sol et dans les premiers horizons entraîne une augmentation progressive de la minéralisation des solutions de la zone non saturée et dans le secteur d'étude 
où la nappe est à moins de $3 \mathrm{~m}$ de profondeur. L'infiltration des eaux de pluies contribue à l'augmentation de la minéralisation des eaux souterraines dans le secteur de Thiaroye/lac Mbeubeuss.

Les moyennes des teneurs en éléments chimiques majeurs des lixiviats des sols de l'aquifère sont supérieures à celles généralement mesurées dans les puits traditionnels et les piézomètres. Cette observation laisse supposer des phénomènes de dilution et/ou de réduction des teneurs ioniques entre la zone non saturée et la nappe. Les mécanismes de migration des substances vers la nappe phréatique sont contrôlés par les flux verticaux et horizontaux liés au lessivage des déchets domestiques de la décharge.

L'étude physico-chimique des eaux souterraines dans la zone du lac Mbeubeuss montre que les eaux souterraines sont moyennement à fortement minéralisées avec des faciès chimiques à dominance chloruré sodique et chloruré calcique. Les fortes teneurs en nitrates mesurées dans les puits villageois situés aux abords de la décharge publique seraient probablement dues au lessivage des produits de la décomposition des ordures ménagères par les eaux de pluies.

\section{References:}

1. Barnes, C.J. \& Allison, G. B. (1983). The distribution of deuterium and oxygen 18 in dry soil: I. Theory. Journal hydrology, 60, pp. 141156.

2. Bellion, Y. J. (1987). Histoire géodynamique post-paléozoïque de l'Afrique de l'Ouest d'après l'étude de quelques bassins sédimentaires (Sénégal, Taoudenni, Iullemmeden, Tchad). Thèse de doctorat d'Etat ès Sciences, Université d'Avignon, France, 302 p.

3. Chamley, H. (2000). Bases de sédimentologie, $1^{\text {er }}$ et $2^{\text {ème }}$ cycles. Edition Dunod (2 ${ }^{\text {ème }}$ ) Paris (France), pp. $34-48$.

4. Cisse Faye, S. (2000). "Nappe libre des sables quaternaires Thiaroye/Beer Thialane. Etude de la contamination par les nitrates sur la base d'un système d'information géographique (PC ARC/INFO)", Thèse de doctorat $3^{\text {ème }}$ cycle, Université CAD, (Sénégal) / IAAG, (Allemagne),

5. Eblin, S.G., Soro, GM., Sombo, A.P., Aka, N., Kambire, O., \& Soro, N. (2014). Hydrochimie des eaux souterraines de la région d'Adiaké (Sud-Est côtier de la cote d'Ivoire). Larhyss Journal, ISSN 1112-3680, $\mathrm{n}^{\circ} 17$, pp. 195-214.

6. Edmunds, W. M. \& Walton, N.R.G. (1980). A geochemical and isotopic approach to recharge evaluation in semi-arid zones: Past and present. Arid Zone hydrology : Investigation with Isotope Techniques, IAEA, Vienne, pp. 47-68. 
7. Essouli, O. F. (2005). Impact de la décharge publique du lac Mbeubeuss sur la ressource en eau de l'aquifère des sables quaternaires de Thiaroye (Dakar, Sénégal). Thèse de doctorat $3^{\text {ème }}$ cycle, Département de Géologie, Faculté des Sciences et Techniques, Université CAD, Dakar, Sénégal, 118 p.

8. Faye, A. (1994). "Recharge et paléorecharge des aquifères profonds du bassin du Sénégal. Apport des isotopes stables et radioactifs de l'environnement et implication paléohydrogéologique et paléoclimatique". Thèse doctorat d'Etat es-Sciences, Université C.A.D., Sénégal.

9. Fidelibus, M. D. \& Tulipano, L. (1996). Regional flow of intruding sea water in the carbonate aquifers of Apulia (Southem Italy). $14^{\text {th }}$ Salt Water Intrusion Meeting. Barcelona, Spain.

10. Garcia, MG., Del, V., Hidalgo, M., \& Blesa, MA. (2001). Geochemistry of groundwater in the alluvial plain of Tucuman province, Argentina. Hydrogeology Journal, vol. 9 (6), pp. 597-610.

11. Gaye, C. B. (1990). Etude isotopique et géochimique du mode de recharge par les pluies et de décharge évaporatoire des aquifères libres sous climat semi-aride au nord du Sénégal. Thèse d'Etat ès Sciences, Département de Géologie, Univ. CAD, Dakar, Sénégal, 245 p.

12. Gouvea Da Silva Rosa, R.B. (1980). Migration des sels et des isotopes lourds à travers des colonnes de sédiment non saturé sous climat semiaride. Thèse $3^{\text {ème }}$ cycle, Univ. Paris VI, France, 116 p.

13. Kouassi, A. M., Yao, A. K., Ake, Y. A., \& Bieme, J. (2011). Application des méthodes d'analyses statistiques multivariées à l'étude de la minéralisation des eaux des aquifères fissurés de la zone Guiglo-Duékoué (Ouest de la cote d'Ivoire). International Journal of Biological and Chemical Sciences, ISSN 1991-8631, 5(5): 2155-2169.

14. Martin, A. (1970). Les nappes de la presqu'île du Cap Vert. Leur utilisation pour l'alimentation en eau de Dakar. Publ. BRGM, 50 p.

15. Mclean, W., Jankowski, J., \& Lavitt, N. (2000). Groundwater quality and sustainability in an alluvial aquifer, Australia. In: Sililo O. et al. (eds). Groundwater, past achievement and future challenges. A. A. Balkema, Rotterdam, pp. 567-573.

16. OMS/WHO (2004). Guidelines for drinking water quality, $3^{\text {nd }}$ edition ISBN 92-4-154638-7, $540 \mathrm{p}$.

17. Tandia, A. A. (2000). Origine, évolution et migration des formes de l'azote minéral dans les aquifères situés sous environnement périurbain non assaini : Cas de la nappe des quaternaires de la région de Dakar (Sénégal). Thèse d'Etat ès Sciences, Département de Géologie, Univ. CAD, Sénégal, 210 p. 
18. Tellam, J. H. (1995). Hydrochemistry of the saline groundwaters of the lower Mersey Basin Permo-Triasssic Sandstone aquifer, UK. Journal of Hydrology, 165, pp. 45-84.

19. Travi, Y., Gac, J.C., \& Fritz, B. (1987). Reconnaissance chimique et isotopique des eaux de pluie au Sénégal. Cahier ORSTOM, pp. 43-53.

20. Zouari, K., Aranyossy, J.F., Mamou, A., \& Fontes, J-Ch. (1984). Etude isotopique et géochimique des mouvements et de l'évolution des solutions de la zone aérée des sols sous climat semi-aride (Sud Tunisien). Publ. IAEA-TECDOC-357, pp. 121-143. 